\title{
TUBERCULOUS LYMPHADENOPATHY IN A CASE OF ERYTHEMA NODOSUM LEPROSUM
}

\author{
Ratan Kr. Kotokey1, Bipul Chandra Kalita², Juma Das ${ }^{3}$, Swarup Kar ${ }^{4}$, Avisek Chakravorty ${ }^{5}$ \\ ${ }_{1}^{1}$ Professor and Principal, Department of Medicine, Assam Medical College and Hospital, Assam. \\ ${ }^{2}$ Associate Professor, Department of Medicine, Assam Medical College and Hospital, Assam. \\ ${ }^{3}$ Assistant Professor, Department of Medicine, Assam Medical College and Hospital, Assam. \\ ${ }_{4}^{4}$ Registrar, Department of Medicine, Assam Medical College and Hospital, Assam. \\ 5Junior Resident, Department of Medicine, Assam Medical College and Hospital, Assam.
}

\section{ABSTRACT}

Erythema nodosum leprosum (ENL) is a debilitating inflammatory complication of leprosy characterised by inflammatory cutaneous nodule with or without ulceration. An intriguing feature of ENL is its exquisite sensitivity to treatment with the immunomodulatory drug thalidomide, which was discovered serendipitously in the early 1960s. Thalidomide is now the treatment of choice for ENL. However, being an immunosuppressant, thalidomide has the potential of making the patient vulnerable to infections. ${ }^{1}$ Tuberculosis (TB) and leprosy are two ancient pathogens, which have been identified as infecting humans since thousands of years. It has been shown from archaeological evidence, post-mortem findings, and retrospective analysis of leprosy institutions' data that there is a highly observed incidence of concomitant infection with leprosy and TB. However, reports of concomitant infection in modern literature remain scarce. Estimation of the annual new case detection rate (ANCDR) in India where both TB (ANCDR 181 per 100,000 in 2011). ${ }^{2}$ and leprosy (ANCDR 10.35 per 100,000 in 2011)..$^{3}$ remain endemic, suggest that only 0.019 cases of concomitant infection per 100,000 population are detected per year.

\section{CASE SUMMARY}

A 30-year-old male was admitted in the Department of Medicine, Assam Medical College and Hospital in September 2015 with complaints of multiple painful swellings over armpits and groins for 20 days along with fever. He had received MDT for leprosy the previous year and was currently on corticosteroids and thalidomide for recurrent ENL reaction. FNAC of the swollen lymph nodes proved them to be of tuberculous origin. The patient was put on ATT under DOTS and he improved eventually.

\section{KEYWORDS}

Tb lymphadenopathy, Recurrent ENL, Thalidomide, Lepra Reaction.

HOW TO CITE THIS ARTICLE: Kotokey RK, Kalita BC, Das J, et al. Tuberculous lymphadenopathy in a case of erythema nodosum leprosum. J. Evolution Med. Dent. Sci. 2016;5(55):3811-3813, DOI: 10.14260/jemds/2016/872

\section{INTRODUCTION}

A 30-year-old man who was already diagnosed to be a case of recurrent ENL reaction and was on thalidomide $100 \mathrm{mg}$ twice daily and prednisolone $40 \mathrm{mg}$ once daily tablets presented with the chief complaints of multiple painful swellings in his armpits and groins for 20 days associated with low-grade fever with evening rise of temperature and malaise and anorexia. He did not have history of cough, expectoration, haemoptysis, pain abdomen, or altered bowel habit. He had a history of being diagnosed with multibacillary leprosy the previous year and had completed the course of MDT for leprosy lasting 9 months. There was no history of contact with any TB cases.

The patient had a dehydrated and toxic look with a coated tongue, fever $\left(101^{*} \mathrm{~F}\right)$, and tachycardia (HR-116/min). There were multiple lymph nodes on his right axilla as well as both groins. The lymph nodes were soft to firm, tender, matted, and mobile. Chest auscultation, heart sounds, and abdominal palpation did not yield any positive findings.

Financial or Other, Competing Interest: None.

Submission 03-06-2016, Peer Review 28-06-2016,

Acceptance 04-07-2016, Published 11-07-2016.

Corresponding Author:

Dr. Avisek Chakravorty,

Room No. 45,

PG Hostel No. 8

Assam Medical College and Hospital,

Assam.

E-mail: avisek.doc@gmail.com

DOI: $10.14260 /$ jemds $/ 2016 / 872$
FNAC of the lymph nodes revealed the cause of lymphadenopathy to be tuberculosis as multiple acid-fast bacilli were seen by ZN staining in the pus aspirated from the LN.

The patient was also found to have sepsis as evidenced by a total leucocyte count of 31,400. His kidney and liver function tests as well as electrolytes and urine examination came out to be normal. There was no abnormality detected in chest $\mathrm{x}$-ray PA view, and his sputum did not test positively for acid-fast bacilli. However, on a high-resolution CT scan of chest, he was found to have patchy ground-glass opacities in the posterobasal segment of lower lobe of the right lung. The patient did not test positive for HIV.

Thus, a diagnosis of tuberculous lymphadenopathy with ENL with CAP was made and the patient was put under Cat.1 ATT under DOTS. Oral prednisolone was gradually tapered and then stopped and the dose of thalidomide was reduced to 100 mg tablet once daily. Within seven days, the patient responded dramatically with resolution of fever and reduction in the size of the lymph nodes. Following his discharge from the hospital, the patient has visited OPD multiple times for follow up. He has had no flares of ENL and has shown persistent improvement as regards the lymphadenopathy and blood parameters. He has now completed 6 months ATT successfully without any major side effect. 


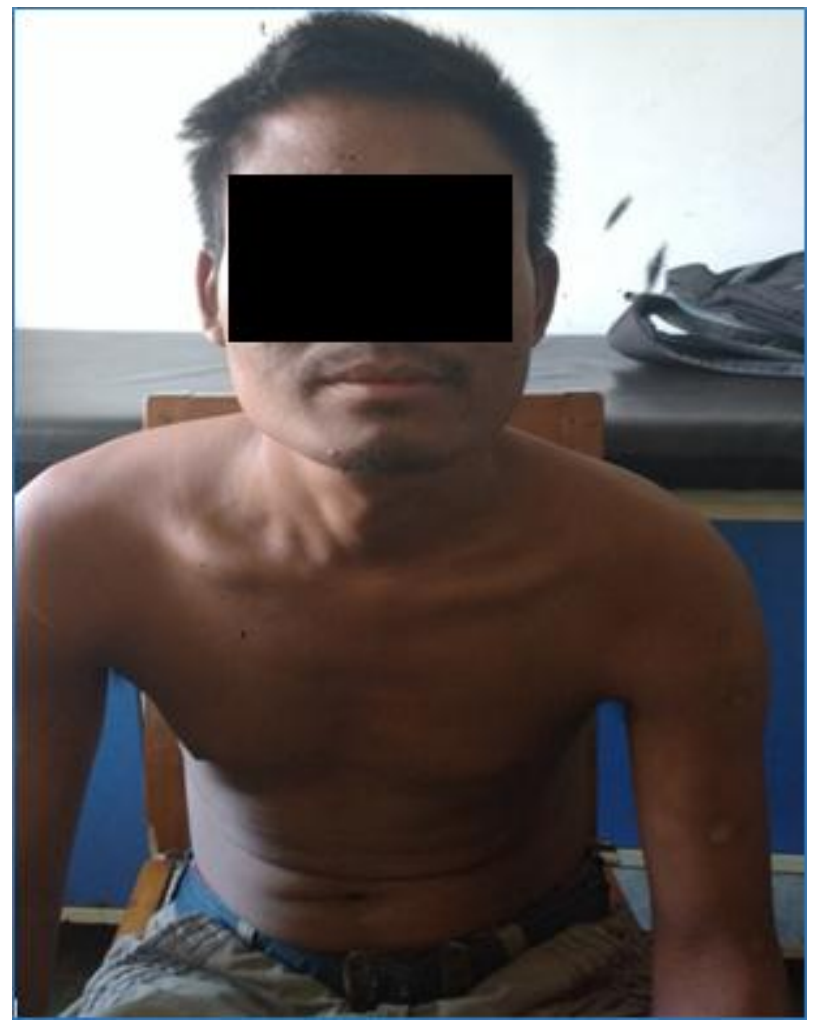

Fig. 1: The Patient

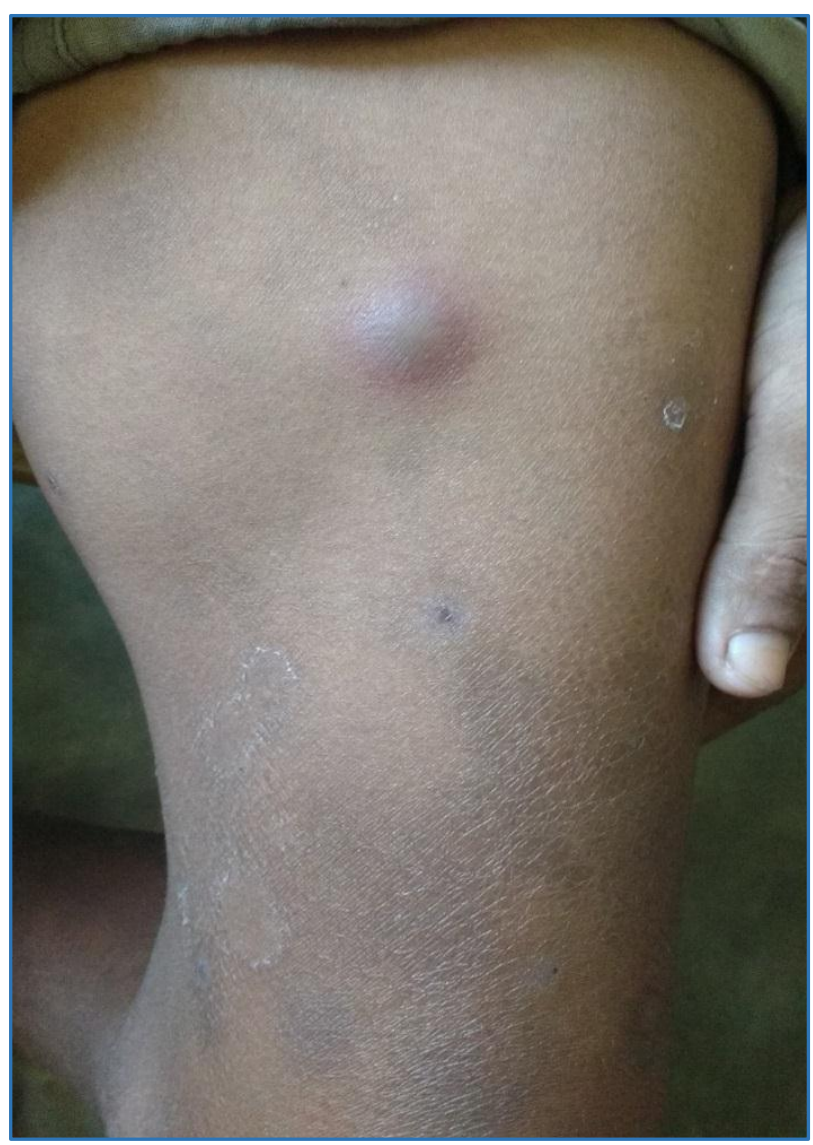

Fig. 2: Inflamed Evanescent ENL Lesion

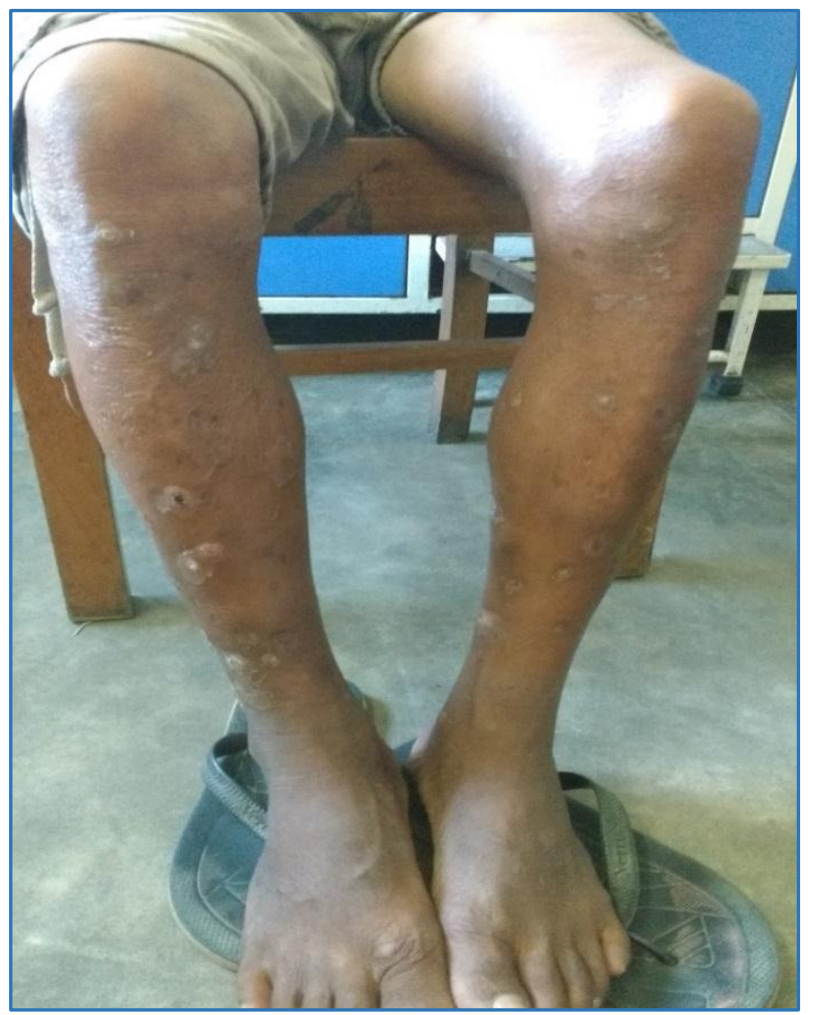

Fig. 3: Stigmata of Recurrent ENL in Both Lower Limbs

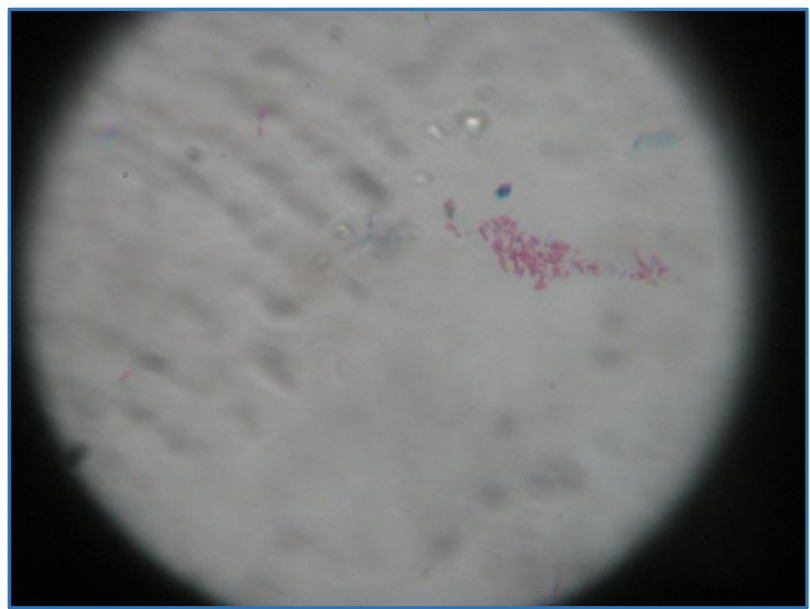

Fig. 4: Clump of Lepra Bacilli

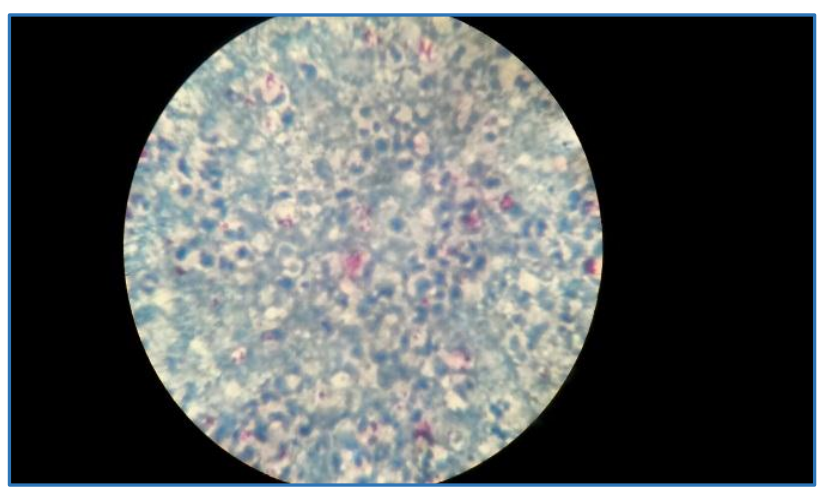

Fig. 5: Acid-Fast Tubercle Bacilli 


\section{DISCUSSION}

The infrequent occurrence of both tuberculosis and leprosy is based on the transmission dynamics of both infections.

The higher reproductive rate of Tubercle bacilli compared to Lepra bacilli and degree of cross immunity within an individual does not allow both infections to occur simultaneously.

First attention about leprosy and tuberculosis co-infection was brought about by Chaussinand in 1948 who concluded that the prevalence of leprosy was inversely related with the prevalence of tuberculosis. ${ }^{4}$ However, in some leprosy communities, tuberculosis appears to be quite common. This may be the result of a small group of people unable to defend themselves against either organism.

The occurrence of leprosy and tuberculosis co-infection was reported for the first time by Relvich in 1954 who strongly argued that association of tuberculoid form of leprosy with tuberculosis was uncommon, but lepromatous forms were more susceptible to develop tuberculosis. ${ }^{5}$ However, Kumar et al in 1982 proved the existence of TB in the entire spectrum of leprosy including tuberculoid, lepromatous, and borderline forms. ${ }^{6}$ Since then, there have been many reports of coinfection. There are reports of triple association of American cutaneous leishmaniasis, lepromatous leprosy, and pulmonary tuberculosis. ${ }^{7}$ There are several case reports of co-infection of HIV and leprosy and also co-infection of leprosy, pulmonary tuberculosis, and cutaneous tuberculosis. ${ }^{8}$ Also, leprosy and TB co-infection has been reported in numerous cases of druginduced immunosuppression.

Any diagnostic confusion regarding the microbiological diagnosis can be resolved with the fact that Ziehl-Neelsen staining of the pus from lymph node was done using $20 \%$ sulphuric acid (H2SO4) under which only tubercle bacilli survive, but not Lepra bacilli. Slit-skin smear was done using $5 \% \mathrm{H} 2 \mathrm{SO} 4$.

\section{CONCLUSION}

It is important to recognise the presence of tuberculosis in leprosy patients so as to avoid a scenario where only a single antitubercular drug is instituted (e.g., rifampicin in MDT of leprosy) as it may contribute to development of acquired drug resistance and reduced effectiveness of anti-TB treatment. All effort should be made to rule out latent tuberculosis before starting steroids in erythema nodosum leprosum patients in order to avoid a flare-up.

\section{REFERENCES}

1. Munjal YP, Sainani GH. (eds). API textbook of medicine. $10^{\text {th }}$ edn. volume 1 , Jaypee brothers publications, $\mathrm{p}-743$.

2. World Health Organisation. Global tuberculosis report 2015.http://www.who.int/tb/publications/global_repor t/en/

3. National Leprosy Eradication Programme. NLEP-progress report for the year 2014-2015. Central leprosy division. nlep.nic.in/pdf/ProgressReport2011-12.pdf.

4. Chaussinand R. Tuberculosis and leprosy-antagonistic illnesses. Int J Lepr 1948;16:431-8.

5. Relvich AL. The treatment of tuberculosis in leprosy patients. Lepr Rev 1954;25(4):179-86.

6. Kumar B, Kaur S, Kataria S, et al. Concomitant occurrence of leprosy and tuberculosis: a clinical, bacteriological and radiological evaluation. Lepr India 1982;54(4):671-6.

7. Delobel P, Launois P, Djossou F, et al. American cutaneous leishmaniasis, lepromatous leprosy, and pulmonary tuberculosis co-infection with down regulation of the Thelper 1 cell response. Clin Infect Dis 2003;37:628-33.

8. Inamadar AC, Sampagavi VV. Concomitant occurrence of leprosy, cutaneous tuberculosis and pulmonary tuberculosis: a case report. Lepr Rev 1994;65(3):282-4. 